\title{
APLICACIÓN DE UN PROGRAMA PSICOEDUCATIVO PARA FOMENTAR LA CREATIVIDAD EN LA ETAPA DE EDUCACIÓN INFANTIL
}

\section{(Applying of a psycho-educative program for developing the creative in the childhood school)}

\author{
por \\ Clemente Franco Justo (다ranco@ual.es) \\ Ficha del artículo \\ $\underline{\text { Sobre los autores }}$ \\ Formato HTML
}

Article record

$\underline{\text { About authors }}$

$\underline{\text { HTML format }}$

\begin{abstract}
The intention of this work is to verify the theoretical assumption which states the relationship between developing creative potential and the the environmental conditions which the context provides. A psychoeducational program based on childrens' stories was designed with the aim of stimulating the creative capability among children in primary school. To measure creativity, the Torrance Test of Creativity Thinking graphic and verbal battery was used. After applying the program, the results showed a huge rise of the levels of verbal creativity in the experimental group with respect to the control groups.
\end{abstract}

\section{Keywords}

Creativity, children's stories, childhood school.

Resumen
En este trabajo, pretendemos comprobar de forma expe-
rimental la asunción teórica que plantea la posibilidad de
fomentar el potencial creativo de acuerdo con las condi-
ciones ambientales que el medio proporciona. Para ello,
se diseñó un programa psicoeducativo basado en cuentos
infantiles, con el fin de estimular la capacidad creativa en
niños de Educación Infantil. Para la medición de la crea-
tividad se utilizaron la Batería Gráfica y Verbal de Test
de Pensamiento Creativo de Torrance. Tras la aplicación
del programa, los resultados arrojaron un incremento sig-
nificativo en los niveles de creatividad verbal en el grupo
experimental respecto a los grupos control.
Descriptores
Creatividad, cuento infantil, educación infantil

factores afectivos, cognitivos y ambientales (Andreucci y Mayo, 1993).

Es indudable la importancia que los factores ambientales juegan en relación con la estimulación o inhibición de la capacidad creativa. De este modo, tanto la familia, como el sistema escolar y la sociedad en gene- 
ral, adquieren gran relevancia en cuanto a su influencia en el grado de la expresión creadora del individuo.

Es por ello que, el sistema educacional, puede ser considerado como el medio más propicio para desarrollar la capacidad creativa, dada la gran cobertura que posee y la gran cantidad de tiempo que los niños pasan allí. Asimismo, la educación tiene por objetivo último el desarrollo integral del hombre, favoreciendo la actualización de todas sus potencialidades. Por este motivo, la estimulación de la capacidad creativa debe ser considerada dentro de los objetivos del sistema educacional.

Madrid (2003), afirma que hay que cultivar la capacidad del sujeto para responder al cambio, desarrollando cualidades críticas de la mente. Para esta autora, esta capacidad se denomina creatividad $\mathrm{y}$, según ella, debe trabajarse fundamentalmente en los años que abarca la Educación Infantil, ya que es en esta edad cuando existe la máxima plasticidad cerebral. Considera, asimismo, que la etapa infantil nos brinda grandes posibilidades para que la espontaneidad creativa de los niños se prolongue el mayor tiempo posible, hasta que tengan una fuente rica de conocimiento activo, que será la base del desarrollo de su capacidad creativa.

Por otra parte, el informe realizado por Delors (1996) para la UNESCO, hace hincapié en que la educación tiene la misión de permitir a todos, sin excepción alguna, hacer fructificar todos sus talentos y todas sus capacidades de creación, lo que implica que cada uno pueda responsabilizarse de sí mismo y realizar su proyecto personal.

En la investigación que aquí se presenta, se considera la creatividad como una capacidad o actitud innata, una característica propia de cada individuo y, como tal, susceptible de ser desarrollada de acuerdo con las experiencias que el medio proporciona. Se considera que dicha capacidad no se manifiesta de igual manera en todos los niños, sino que está re- lacionada con el hecho de que hay algunos ambientes que limitan la expresión de la creatividad, y otros que la favorecen.

Al ser la creatividad una característica innata, presente en todos los individuos y al ser una potencialidad susceptible de desarrollo en función de las condiciones ambientales, su estimulación es particularmente útil en el nivel de Educación Infantil, porque el niño está en formación y, si proviene de un hogar en donde no se le ha estimulado, es el momento oportuno para contrarrestar esas influencias negativas.

Por lo tanto, el hecho de que algunos niños sean naturalmente creativos no significa que no puedan serlo aún más si se les somete a cierto entrenamiento y estimulación mediante las técnicas adecuadas. Y tampoco significa que otros niños no puedan llegar a ser creativos, ya que, aprender a pensar y a actuar creativamente puede ser aprendido de la misma forma que se aprende en otras áreas del saber (Gadner, 1999).

Normalmente, en estos casos, no nos quedamos cruzados de mano y afirmamos que el talento natural es suficiente, sino que sabemos que podemos entrenar a los niños para que alcancen cierto nivel de competencia, ya que el talento natural, si existe, se verá favorecido por el entrenamiento y la estimulación mediante las técnicas adecuadas.

Se ha comprobado que las técnicas más exitosas para estimular la creatividad son las que abarcan tanto los procesos cognitivos, como afectivos (Moore, Ugarte, Urrutia 1987). En este sentido, el cuento infantil abarca ambas dimensiones, impacto afectivo y elementos cognitivos, al favorecer la imaginación y permitiendo una total divergencia del pensamiento.

Tal como plantean varios autores (Andreucci y Mayo, 1993, Rodari, 1996), la gran mayoría de los programas infantiles que aparecen en televisión son eminentemente pasivos, donde se deja muy poco espacio al niño 
para que interactúe, piense, cree e imagine, ya que la imagen se le entrega hecha. Es por ello, que el cuento puede ejercer un papel protector contra la pasividad antinatural a la que el niño se ve sometido hoy día. El cuento permite al niño interpretar desde su mundo interior el mundo que le rodea, proporcionándole una gran cantidad de material novedoso y fascinante, que le permite enriquecer, de este modo, su mundo imaginario y su actividad lúdica, elementos indispensables para el desarrollo del potencial creador en la infancia, ya que a través de ellos (imaginación y juego), el niño desarrolla, canaliza y expresa su creatividad. Los niños crean a partir de las palabras que escuchan en los cuentos sus propias imágenes mentales, constituyendo, de esta manera, una herramienta útil para el desarrollo de la creatividad infantil.

El niño es creador por naturaleza, y es por ello, que el cuento se nos presenta como un elemento indispensable para canalizar dicho potencial creativo. A través del cuento, el niño puede desarrollar sus capacidades de fluidez, flexibilidad y originalidad, imaginando un final distinto, introduciendo y eliminando personajes, modificando la trama y los diálogos, escenificándolo, etc. Es decir, dándole la oportunidad de jugar y de crear con y, a partir del cuento.

Por tanto, el principal objetivo de la presente investigación, fue demostrar la factibilidad de estimular la capacidad creativa de los niños a través del sistema educacional mediante el empleo de cuentos tradicionales evaluando experimentalmente un programa para el desarrollo de esta capacidad.

Como hipótesis general, tenemos que la capacidad creativa puede ser estimulada a través de la implementación de un programa psicoeducativo, basado en cinco cuentos tradicionales, especialmente diseñado para ello.

A partir de dicha hipótesis general, se desprenden las hipótesis específicas que guían esta investigación:
1. Aquellos niños sometidos al programa de intervención (grupo experimental), van a manifestar un incremento significativamente mayor, en la expresión de la creatividad verbal, medida en sus aspectos de fluidez, flexibilidad y originalidad, que aquellos niños no sometidos al programa (grupos control).

2. Aquellos niños sometidos al programa de intervención (grupo experimental), van a manifestar un incremento significativamente mayor, en la expresión de la creatividad gráfica, medida en sus aspectos de fluidez, flexibilidad y originalidad, que aquellos niños no sometidos al programa (grupos control).

\section{METODOLOGÍA}

\subsection{Sujetos}

La muestra estuvo constituida por 63 niños, 23 de ellos pertenecientes al grupo experimental (11 niños y 12 niñas), 21 correspondientes al primer grupo control (13 niños y 8 niñas) y 19 al segundo grupo control (8 niños y 11 niñas). Las edades de los niños oscilaban entre los 5 años cero meses, y los 5 años 11 meses y contaban con un rango intelectual normal promedio como mínimo.

La variable capacidad intelectual se consideró para controlar la posible intervención de este factor en el desarrollo de la creatividad de los niños, ya que, autores como Rieben (1979), establecen que ambos aspectos (creatividad e inteligencia), se encuentran relacionados entre sí, afirmando que se requeriría como mínimo de un rango normal de capacidad intelectual para desarrollar plenamente el potencial creativo. Por lo tanto, para controlar la intervención de esta variable, se estableció como criterio que los niños de la muestra tuvieran como mínimo un rendimiento intelectual correspondiente a un rango normal promedio, CI $>90$, ya que éste es el CI mínimo necesario que establece Repucci (1980), para que pueda manifestarse la creatividad (Moore, Ugarte y Urrutia,1987). 
Para los efectos de mortalidad experimental, se tomaron grupos naturales de niños asistentes la semana de aplicación del primer pretest.

\subsection{Instrumentos}

Para la evaluación de la capacidad intelectual general de los niños se utilizó el Test de Matrices Progresivas de Raven. Este instrumento es de carácter no verbal y evalúa la capacidad intelectual general de los niños entre los 5 y 12 años a través del factor visoespecial.

Debido a que el test de Raven posee una alta saturación del factor visoespacial, y para descartar la influencia de problemas visoperceptivos en el rendimiento en dicho test, se aplicó la Escala de Inteligencia de Wechsler para Preescolar y Primaria (WPPSI) a aquellos niños cuya puntuación en el Test de Raven correspondiera a un percentil inferior a 50 , siguiendo las recomendaciones planteadas por Moore, Ugarte y Urrutia (1987).

Para la evaluación de la creatividad gráfica se utilizó la Batería Gráfica del Test de Pensamiento Creativo de Torrance (1974).

Para la evaluación de la creatividad verbal se utilizó la Batería Verbal del Test de Pensamiento Creativo de Torrance (1974).

Se utilizó la forma A de dichas pruebas en las fases pretest y la forma B en la postest, tal y como sugiere el autor, valorándose la fluidez, flexibilidad y originalidad de acuerdo a los siguientes criterios:

- Fluidez: se concede un punto por cada idea presentada, sin tomar en consideración las repetidas y las no relevantes (en cuanto que no cumplen los requisitos de la tarea específica)

- Flexibilidad: se valora el número de categorías o agrupamientos temáticos posibles de las ideas, otorgando un punto por cada categoría empleada. Las diferentes categorías vienen detalladas en el manual para cada una de las actividades.
- Originalidad: se puntúa en función de la infrecuencia estadística de la idea, de acuerdo a los siguientes criterios:

a) 0 puntos si la idea está indicada por más de tres sujetos

b) 1 punto si la idea está planteada por tres sujetos

c) 2 puntos si la idea ha sido propuesta o repetida por dos sujetos

d) 3 puntos si la idea señalada aparece en un sujeto

\subsection{Procedimiento}

Para lograr los objetivos planteados en este estudio, se utilizó un diseño cuasiexperimental de comparación de grupos, con medición pretest-pretest-postest, con un grupo experimental y dos grupos control. Dicha medición se realizó con la finalidad de explorar los efectos del programa (variable independiente), en variables dependientes tales como la creatividad verbal y la creatividad gráfica.

Al no tratarse de un diseño experimental, se intentó incrementar la validez interna del estudio, para lo cual se utilizó un diseño con grupos de control no equivalentes con doble pretest, utilizándose dos grupos control, y realizándose dos medidas pretest (esto último con el fin de evaluar el efecto diferencial que la variable maduración pudiera tener en el estudio). Asimismo, para aumentar este control del factor madurativo, el tiempo transcurrido entre el primer y el segundo pretest, $\mathrm{y}$ entre el segundo pretest y el postest fue el mismo (11 semanas), tal y como aconsejan Anguera y otros (1995).

Igualmente, se aplicó una técnica de triple ciego, ya que los niños no conocían el tratamiento, ninguna de las profesoras conocía el objetivo de la investigación (desconociendo la existencia de otros grupos en la investigación), y los evaluadores no conocían la pertenencia de los sujetos a los grupos experimental o control.

El objetivo de la evaluación inicial fue conocer el nivel básico de creatividad de los niños de los tres grupos. Para ello se admi- 
nistro de forma individual, en el mismo colegio el "Torrance Test of Creative Thinking" en su forma A.

Asimismo, se aplicó el test de Matrices Progresivas de Raven, de forma individual en el colegio, con el fin de obtener una estimación del rango intelectual de los sujetos participantes en la investigación.

Tan solo dos sujetos del grupo experimental mostraron un percentil inferior a 50, razón por la cual se les aplicó el WPPSI para descartar posibles problemas visoperceptivos. Tras la realización de esta prueba y tras constatar que su puntuación era inferior a 90 se decidió excluir a dichos sujetos a la hora de tomar en cuenta sus datos para el análisis estadístico, aunque sí recibieron el programa de intervención.

Para controlar el posible efecto que la variable evaluador podía tener en los resultados obtenidos, cuatro semanas antes de realizarse esta primera evaluación, los evaluadores se incorporaron a las clases a las cuales evaluaron. Fueron presentados como profesores en prácticas tomando durante este período contacto con los niños, ya que según Marín (1980), es necesario en la evaluación de la creatividad la existencia de un clima efectivo de confianza y aceptación, ya que la existencia de un ambiente evaluativo inhibiría la manifestación de respuestas creativas.

Once semanas después de esta primera medición, y para controlar la posible incidencia del factor madurativo, se realizó otra medición pretest de todos los sujetos con la forma A del TTCT.

Una vez finalizada esta evaluación preexperimental, se procedió a la aplicación del programa en el grupo experimental.

El propósito de la intervención consistió en crear un programa compuesto por 20 actividades en base a cinco cuentos tradicionales (Cenicienta, Bambi, La casita de chocolate, Blancanieves y Pinocho), con la intención de estimular o potenciar tres dimensiones de la capacidad creativa en los niños de Educación Infantil: fluidez, flexibilidad y originalidad.

El programa tuvo una duración de 10 semanas y estuvo constituido por 20 sesiones (cuatro sesiones por cuento). Cada actividad duró entre 45 y 70 minutos y fueron realizadas dos veces a la semana. La duración del programa fue de tan sólo diez semanas con el fin de aminorar los riesgos de maduración. Se tuvo presente, asimismo, que a mayor tiempo transcurrido entre el pretest y el postest, aumenta la probabilidad de interferencia de otras variables ajenas a los programas sometidos a prueba. Además, tal y como establecen Anguera y otros (1995), el diseño pretest-postest no permite argumentos válidos de causalidad a menos que se trabaje con cortos o muy cortos intervalos de tiempo entre pretest y postest, y por tanto, no resulta recomendable en situaciones donde se evalúa una intervención psicológica o educativa a largo plazo

Los objetivos de las actividades fueron favorecer las tres dimensiones de la creatividad antes mencionadas, principalmente, a través de tres criterios básicos para desarrollar el pensamiento divergente (Andreucci y Mayo, 1993):

\section{Usos inusuales \\ 2. Mejora del objeto \\ 3. Supongamos que}

A modo de ejemplo, se presenta una sesión en el Anexo 1 en la cual puede observarse el planteamiento y estructura de las distintas sesiones que componen el programa de intervención.

Una vez finalizada la aplicación del programa diseñado, se realizó la evaluación postest a los tres grupos, mediante la forma B del TTCT. El objetivo de esta evaluación fue constatar el efecto de la aplicación del programa en la estimulación de la creatividad a través del cuento y de las actividades derivadas de éstos. 
Cada uno de los tests que evaluaban la creatividad fue corregido por cuatro evaluadores con el fin de obtener una medición lo más objetiva posible (fiabilidad interjueces). Asimismo, para aumentar la validez y fiabilidad de las correcciones, se les asignó un código a los niños, con el fin de que a la hora de corregir los tests no se supiese si pertenecían al grupo experimental o a los grupos control.

\section{RESULTADOS}

Para analizar los resultados de la evaluación de la creatividad (área verbal y gráfica), se efectuó una comparación de promedios por medio de un análisis de covarianza (ANCOVA), con el objetivo de evaluar el cambio producido por el programa de intervención en las variables estudiadas. Para dicho análisis se utilizó el programa estadístico SPSS, versión 11.0.

Se realizó un ANCOVA de cada variable en el postest tomando como covariada su equivalente en el segundo pretest, que indicaría los niveles previos de la variable antes de aplicar el tratamiento al grupo experimental. Con esto se pretende eliminar la influencia que puedan tener los niveles previos en los resultados del postest.

Se ha utilizado un análisis de covarianza como técnica de análisis de los datos obtenidos, ya que es una técnica recomendada cuando se tiene un reducido número de sujetos o cuando se han utilizado grupos naturales (García Jiménez, 1992). Además, según García, Frías y Pascual (1995), es la mejor técnica de análisis de datos que se conoce para los diseños con grupos de control no equivalentes y doble pretest.

También se realizó un ANOVA de cada variable en el postest, que analizaría la existencia o no de diferencias significativas entre los grupos sin tener en cuenta los niveles de creatividad de partida. Los resultados de este análisis hemos de interpretarlos siempre a la luz de los obtenidos en el ANCOVA, ya que podemos obtener diferencias significativas que achaquemos al experimento y sean simplemente debidas a los distintos niveles previos de creatividad de los que partían los sujetos del estudio; y por el contrario, los efectos del experimento podrían verse camuflados si las ganancias obtenidas por los distintos grupos acaban igualando los resultados finales por partir los sujetos de niveles previos de creatividad diferentes.

Asimismo, se realizó un test de rangos múltiples o test de comparaciones múltiples, con el objetivo de detectar entre que grupos existen diferencias. Dicho test no hubiese tenido cabida en este estudio si hubiésemos trabajado sólo con dos grupos, pero en nuestro caso ha sido necesario para confirmar que las diferencias encontradas son siempre entre cada uno de los grupos control con el grupo experimental y no entre los grupos control entre sí.

Por último, se realizó un test de muestras apareadas, con el fin de comparar las medias del grupo experimental en los tres momentos de la medición (pretest 1 , pretest 2 y postest). $\mathrm{Si}$ el tratamiento ha sido efectivo deberían encontrarse diferencias significativas entre el segundo prestest y el postest y no así entre los dos pretest.

Antes de comenzar con la exposición de los resultados obtenidos, hay que aclarar la notación empleada para hacer referencia a las variables y medidas obtenidas. Las primeras letras indican la variable analizada: Flexibilidad (Fle), Fluidez (Flu) y Originalidad (O). La letra entre guiones se refiere a si esta variable es Verbal (V), o Gráfica (M). La última letra es para indicar el momento de la medición: primer pretest $(\mathrm{X})$, segundo pretest $(\mathrm{Y})$, y puntuación postest $(\mathrm{Z})$. Al realizar el Test de Muestras Apareadas en el Grupo Experimental, Par 1 indica la comparación de las medias muestrales entre las dos puntuaciones pretest, y Par 2 indica la comparación de las medias muestrales entre el segundo pretest y la puntuación postest. 
Franco Justo, C. (2004). Aplicación de un programa psicoeducativo para formentar la creatividad en la etapa de educación infantil. RELIEVE, v. 10, n. 2, p. 245-266.

http://www.uv.es/RELIEVE/v10n2/RELIEVEv10n2 4.htm

\section{Creatividad Verbal}

\begin{tabular}{|c|c|c|c|c|c|}
\hline VARIABLE & "GRUPO & MEDIDA & 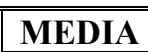 & D.T. & $\begin{array}{l}\text { C.V. } \\
\text {. }\end{array}$ \\
\hline \multirow{9}{*}{ Flexibilidad } & \multirow{3}{*}{ Control 1} & Pretest 1 & 13.48 & 2.994 & 0.222 \\
\hline & & Pretest 2 & 14.14 & 3.198 & 0.226 \\
\hline & & Postest & 14.57 & 3.108 & 0.213 \\
\hline & \multirow{3}{*}{ Control 2} & Pretest 1 & 13.21 & 3.119 & 0.236 \\
\hline & & Pretest 2 & 13.47 & 2.568 & 0.191 \\
\hline & & Postest & 14.11 & 2.787 & 0.198 \\
\hline & \multirow{3}{*}{ Experimental } & Pretest 1 & 13.04 & 3.169 & 0.243 \\
\hline & & Pretest 2 & 15.17 & 4.569 & 0.301 \\
\hline & & Postest & 21.22 & 3.014 & 0.142 \\
\hline \multirow{9}{*}{ Fluidez } & \multirow{3}{*}{ Control 1} & $\begin{array}{l}\text { Pretest } 1 \\
\end{array}$ & 19.29 & 4.101 & 0.213 \\
\hline & & Pretest 2 & 19.95 & 4.566 & 0.229 \\
\hline & & Postest & 20.52 & 4.523 & 0.220 \\
\hline & \multirow{3}{*}{ Control 2} & Pretest 1 & 18.47 & 3.642 & 0.197 \\
\hline & & Pretest 2 & 19.05 & 4.020 & 0.211 \\
\hline & & Postest & 19.21 & 4.354 & 0.227 \\
\hline & \multirow{3}{*}{ Experimental } & Pretest 1 & 20.35 & 5.158 & 0.253 \\
\hline & & Pretest 2 & 21.13 & 4.751 & 0.225 \\
\hline & & Postest & 35.83 & 7.234 & 0.202 \\
\hline \multirow{9}{*}{ Originalidad } & \multirow{3}{*}{ Control 1} & Pretest 1 & 20.38 & 3.892 & 0.191 \\
\hline & & Pretest 2 & 20.43 & 4.214 & 0.206 \\
\hline & & Postest & 20.86 & 3.941 & 0.189 \\
\hline & \multirow{3}{*}{ Control 2} & Pretest 1 & 19.32 & 3.318 & 0.172 \\
\hline & & Pretest 2 & 19.68 & 3.560 & 0.181 \\
\hline & & Postest & 19.53 & 4.274 & 0.219 \\
\hline & \multirow{3}{*}{ Experimental } & Pretest 1 & 21.35 & 5.565 & 0.261 \\
\hline & & Pretest 2 & 21.65 & 5.280 & 0.244 \\
\hline & & Postest & 40.91 & 7.669 & 0.187 \\
\hline
\end{tabular}

Dado que tanto la media aritmética como la desviación típica son valores absolutos, se ha obtenido también el Coeficiente de Variación que nos proporciona una medida relativa de la dispersión. El coeficiente de variación no es más que el cociente entre desviación típica y media. Cuanto menor sea este coeficiente menor será la dispersión de los datos.

En cuanto al aspecto verbal de la creatividad vemos que esta dispersión no es muy alta, oscilando entre un valor de 0.142 que presenta el grupo experimental en la medida prestest de la variable flexibilidad verbal y
0.301 del mismo grupo y la misma variable en la segunda medida pretest.

Se aprecian también a primera vista medias más altas en el grupo experimental que en los grupos control.

\section{Estudio de la variable: Flexibilidad Verbal}

Se han obtenido diferencias significativas entre los grupos control y el grupo experimental ( $\mathrm{F}=58,684 ; \mathrm{p}<0.001$ ), así como también entre la segunda medida pretest y la postest para el grupo experimental $(\mathrm{t}=$ 7.341; $<<0.001$ ). 
Franco Justo, C. (2004). Aplicación de un programa psicoeducativo para formentar la creatividad en la etapa de educación infantil. RELIEVE, v. 10, n. 2, p. 245-266.

http://www.uv.es/RELIEVE/v10n2/RELIEVEv10n2 4.htm

Tabla 2. Análisis de Covarianza

\begin{tabular}{|l||c||c||c||c||c||}
\hline \multicolumn{1}{|c||}{ Fuente } & Suma de Cuadrados & gl & $\begin{array}{c}\text { Desviaciones } \\
\text { dias }\end{array}$ & F & Sig. \\
\hline \hline Modelo Corregido & 961,797 & 3 & 320,599 & 72,219 &, 000 \\
\hline Constante & 246,016 & 1 & 246,016 & 55,418 &, 000 \\
\hline GRUPO & $\mathbf{5 2 1 , 0 3 0}$ & $\mathbf{2}$ & $\mathbf{2 6 0 , 5 1 5}$ & $\mathbf{5 8 , 6 8 4}$ & $\mathbf{, 0 0 0}$ \\
\hline FLE_V_Y & 270,928 & 1 & 270,928 & 61,03 &, 000 \\
\hline Error & 261,918 & 59 & 4,439 & & \\
\hline Total & 19126,000 & 63 & & & \\
\hline Total Corregido & 1223,714 & 62 & & \\
\hline \hline
\end{tabular}

Esta tabla muestra que las diferencias entre con un p-valor menor que una milésima. grupos en el postest son significaticas,

Tabla 3. ANOVA de las ganancias $2^{\circ}$ pretest-postest:

\begin{tabular}{|c|c|c|c|c|c|}
\hline Fuente & Suma de Cuadrados & $\mathrm{gl}$ & \begin{tabular}{|c|} 
Desviaciones Cua- \\
dráticas Medias \\
\end{tabular} & $\mathrm{F}$ & Sig. \\
\hline Modelo Corregido & 690,869 & 2 & 345,434 & 38,897 & ,000 \\
\hline Constante & 17320,245 & 1 & 17320,245 & 1950,312 & ,000 \\
\hline GRUPO & 690,869 & 2 & 345,434 & $\mathbf{3 8 , 8 9 7}$ &, 000 \\
\hline Error & 532,845 & 60 & 8,881 & & \\
\hline Total & 19126,000 & 63 & & & \\
\hline Total Coregido & 1223,714 & 62 & & & \\
\hline
\end{tabular}

Se confirman las diferencias significativas entre grupos.

Tabla 4. Test de comparaciones múltiples:

\begin{tabular}{|c|c|c|c|c|c|c|c|}
\hline \multirow{8}{*}{ Scheffe } & \multirow[b]{2}{*}{ (I) GRUPO } & \multirow[b]{2}{*}{ (J) GRUPO } & \multirow[b]{2}{*}{\begin{tabular}{|c|} 
Diferencia de \\
Medias (I-J)
\end{tabular}} & \multirow[b]{2}{*}{\begin{tabular}{|c} 
Error Están- \\
dar
\end{tabular}} & \multirow[b]{2}{*}{ Sig. } & \multicolumn{2}{|c|}{ 95\% Intervalo de Confianza } \\
\hline & & & & & & $\begin{array}{l}\text { Extremo Infe- } \\
\text { rior } \\
\end{array}$ & $\begin{array}{c}\text { Extremo Supe- } \\
\text { rior }\end{array}$ \\
\hline & \multirow[t]{2}{*}{ c1 } & $\mathrm{C} 2$ & , 47 & (2,899 & (874 & $-1,79$ & 2,73 \\
\hline & & $\mathbf{E}$ & $-6,65$ &, 857 &, 000 & $-8,80$ & $-4,49$ \\
\hline & \multirow[t]{2}{*}{ c2 } & $\mathrm{C} 1$ &,- 47 & ,899 & ,874 & $-2,73$ & 1,79 \\
\hline & & $\mathbf{E}$ & $-7,11$ & ,880 &, 000 & $-9,32$ & $-4,90$ \\
\hline & \multirow[t]{2}{*}{ e } & C1 & 6,65 & ,857 &, 000 & 4,49 & 8,80 \\
\hline & & $\mathrm{C2}$ & 7,11 &, 880 &, 000 & 4,90 & 9,32 \\
\hline
\end{tabular}

Como vemos en la tabla el test de rangos múltiples considerado encuentra diferencias significativas al $99 \%$ entre el grupo experi- mental y ambos grupos control; mientras que no hay evidencias para descartar la hipótesis de igualdad entre los 2 grupos control. 


\section{EVOLUCIÓN DE LA FLEXIBILIDAC}

\section{EN LOS DISTINTOS GRUPOS}

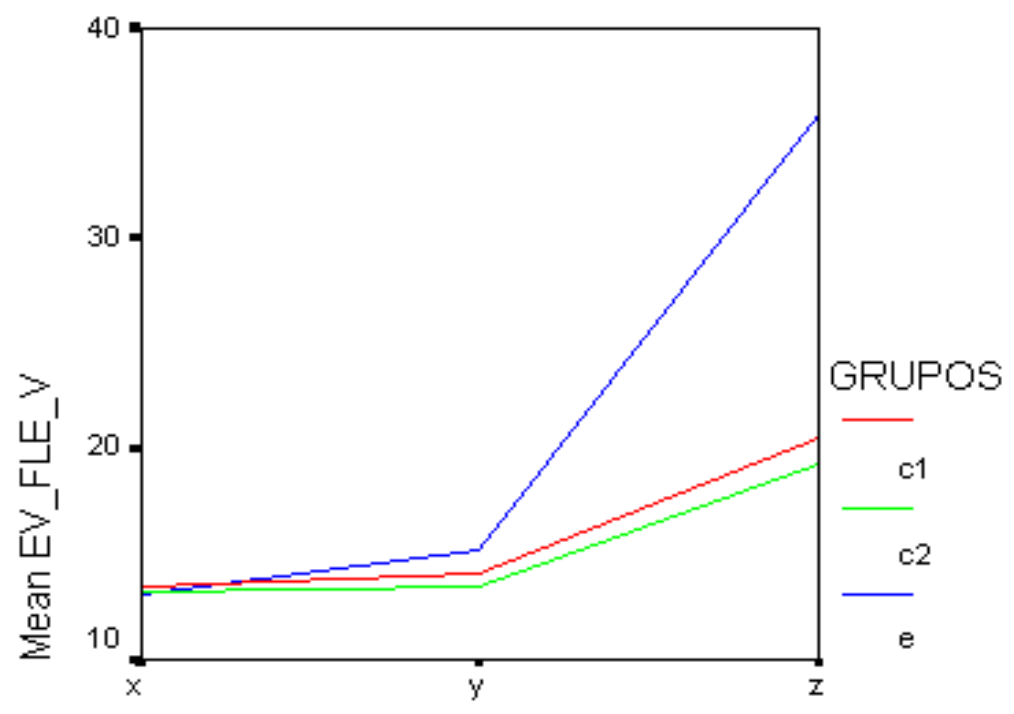

PERIODOS

Para comprobar si existían diferencias significativas entre las medias del grupo experimental en los distintos momentos de medi- ción, realizamos un test de comparación de medias para muestras apareadas, obteniendo los siguientes resultados:

Tabla 5

\begin{tabular}{|c|c|c|c|c|c|c|c|c|}
\hline & \multirow{3}{*}{ Media } & \multirow{3}{*}{$\begin{array}{c}\text { Desviación } \\
\text { Típica }\end{array}$} & \multirow{3}{*}{$\begin{array}{c}\text { Error } \\
\text { Estándar }\end{array}$} & & & \multirow{3}{*}{$\mathrm{t}$} & \multirow{3}{*}{$\mathrm{gl}$} & \multirow{3}{*}{$\begin{array}{l}\text { Nivel de Signi- } \\
\text { ficación a } 2 \\
\text { Colas }\end{array}$} \\
\hline & & & & \multicolumn{2}{|c|}{$\begin{array}{c}95 \% \text { Intervalo de } \\
\text { Confianza }\end{array}$} & & & \\
\hline & & & & Inferior & Superior & & & \\
\hline Par 1 &,- 7826 & 2,50375 &, 52207 & -18653 &, 3001 & $-1,499$ & 22 & 148 \\
\hline Par 2 & 14,6957 & 6,09347 & 1,27058 & 17,330 & 12,06 & $-11,56$ & 22 &, 000 \\
\hline
\end{tabular}

Como vemos el segundo intervalo de confianza para la diferencia de medias no incluye al cero por lo que podríamos concluir que existen diferencias en este par de datos. Vemos a continuación que el valor del estadístico de contraste es significativo por lo que hay evidencias claras de que las medias no son iguales. Para el primer par ocurre al contrario, las diferencias entre los datos obtenidos en los dos pretests no son significativamente distintas de cero, por lo que se han detectado diferencias significativas entre el segundo pretest y el postest para el grupo experimental.

\section{Estudio de la variable: Fluidez Verbal}

Para esta variable hemos obtenido diferencias significativas entre los distintos grupos en el postest ( $\mathrm{F}=99,963$; $\mathrm{p}<0.001$ ), así como entre el segundo pretest y el postest para el grupo experimental $(\mathrm{t}=-11.566 ; \mathrm{p}<$ $0.001)$. 
Franco Justo, C. (2004). Aplicación de un programa psicoeducativo para formentar la creatividad en la etapa de educación infantil. RELIEVE, v. 10, n. 2, p. 245-266.

http://www.uv.es/RELIEVE/v10n2/RELIEVEv10n2 4.htm

Tabla 6. ANCOVA

\begin{tabular}{|l||c|c|c|c|c||}
\hline \multicolumn{1}{|c||}{ Fuente } & Suma de Cuadrados & gl & $\begin{array}{c}\text { Desviaciones Cua- } \\
\text { dráticas Medias }\end{array}$ & F & Sig. \\
\hline \hline Modelo Corregido & 4751,144 & 3 & 1583,715 & 107,190 &, 000 \\
\hline Constante & 126,534 & 1 & 126,534 & 8,564 &, 005 \\
\hline GRUPO & $\mathbf{2 9 5 3 , 8 6 3}$ & $\mathbf{2}$ & $\mathbf{1 4 7 6 , 9 3 1}$ & $\mathbf{9 9 , 9 6 3}$ & $\mathbf{, 0 0 0}$ \\
\hline FLU_V_Y & 1029,987 & 1 & 1029,987 & 69,712 &, 000 \\
\hline Error & 871,713 & 59 & 14,775 & & \\
\hline Total & 47280,000 & 63 & & & \\
\hline Total Corregido & 5622,857 & 62 & & & \\
\hline
\end{tabular}

$R$ Cuadrado $=, 845$ (R Cuadrado Ajustado $=, 837$ )

En negrita vemos como la diferencia entre grupos en la medición postest de la fluidez verbal resulta significativa.

Tabla 7. ANOVA

\begin{tabular}{|l||c||c||c||c||c||}
\hline \multicolumn{1}{|c||}{ Fuente } & Suma de Cuadrados & gl & $\begin{array}{c}\text { Desviaciones Cua- } \\
\text { dráticas Medias }\end{array}$ & F & Sig. \\
\hline \hline Modelo Corregido & 3721,157 & 2 & 1860,578 & 58,703 &, 000 \\
\hline Constante & 39723,243 & 1 & 39723,243 & 1253,297 &, 000 \\
\hline GRUPO & $\mathbf{3 7 2 1 , 1 5 7}$ & $\mathbf{2}$ & $\mathbf{1 8 6 0 , 5 7 8}$ & $\mathbf{5 8 , 7 0 4}$ & $\mathbf{0 0 0}$ \\
\hline Error & 1901,700 & 60 & 31,695 & & \\
\hline Total & 47280,000 & 63 & & & \\
\hline Total Corregido & 5622,857 & 62 & & & \\
\hline \hline
\end{tabular}

$R$ Cuadrado $=, 662$ ( $R$ Cuadrado Ajustado $=, 651)$

Se confirman las diferencias significativas entre grupos.

Al igual que pasaba con la flexibilidad verbal, el test de rangos múltiples utilizado confirma la existencia de diferencias signifi- cativas entre el grupo experimental y los dos grupos control, mientras que no las hay entre ambos grupos control (ver tabla 8).

Tabla 8.

\begin{tabular}{|c|c|c|c|c|c|c|c|}
\hline \multirow{8}{*}{ Scheffe } & \multirow[b]{2}{*}{ (I) GRUPC } & \multirow[b]{2}{*}{ (J) GRUPO } & \multirow[b]{2}{*}{\begin{tabular}{|c|} 
Diferencia \\
de Medias \\
$(\mathrm{I}-\mathrm{J})$
\end{tabular}} & \multirow[b]{2}{*}{ Error Estándar } & \multirow[b]{2}{*}{ Sig. } & \multicolumn{2}{|c|}{$95 \%$ Intervalo de Confianza } \\
\hline & & & & & & $\begin{array}{l}\text { Extremo } \\
\text { Inferior }\end{array}$ & $\begin{array}{l}\text { Extremo } \\
\text { Superior }\end{array}$ \\
\hline & \multirow[t]{2}{*}{$\mathrm{c} 1$} & "C2 & 1,31 & 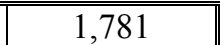 & ",763 & $-3,16$ & 5,79 \\
\hline & & $\mathrm{E}$ & $-15,30$ & 1,697 &, 000 & $-19,57$ & $-11,04$ \\
\hline & \multirow[t]{2}{*}{$\mathrm{c} 2$} & $\mathrm{C} 1$ & $-1,31$ & 1,781 &, 763 & $-5,79$ & 3,16 \\
\hline & & $\mathrm{E}$ & $-16,62$ & 1,743 &, 000 & $-21,00$ & $-12,23$ \\
\hline & \multirow[t]{2}{*}{$\mathrm{E}$} & $\mathrm{C} 1$ & 15,30 & 1,697 &, 000 & 11,04 & 19,57 \\
\hline & & $\mathrm{C} 2$ & 16,62 & 1,743 &, 000 & 12,23 & 21,00 \\
\hline
\end{tabular}

Al igual que en la variable anterior, vemos en la tabla que el test de rangos múltiples considerado encuentra diferencias significativas al 99\% entre el grupo experimental y ambos grupos control; mientras que no hay evidencias para descartar la hipótesis de igualdad entre los 2 grupos control.

Al analizar solamente los datos del grupo experimental en los tres periodos de medi- 
Franco Justo, C. (2004). Aplicación de un programa psicoeducativo para formentar la creatividad en la etapa de educación infantil. RELIEVE, v. 10, n. 2, p. 245-266.

http://www.uv.es/RELIEVE/v10n2/RELIEVEv10n2 4.htm

ción mediante un contraste de medias para muestras apareadas, obtuvimos los siguientes

resultados:

Tabla 9.

\begin{tabular}{|c|c|c|c|c|c|c|c|c|}
\hline & \multirow{3}{*}{ Media } & \multirow{3}{*}{$\begin{array}{l}\text { Desviación } \\
\text { Típica }\end{array}$} & \multirow{3}{*}{$\begin{array}{l}\text { Error } \\
\text { Estándar }\end{array}$} & & & \multirow{3}{*}{$\mathrm{t}$} & \multirow{3}{*}{ gl } & \multirow{3}{*}{$\begin{array}{l}\text { Nivel de Sig- } \\
\text { nificación a } 2 \\
\text { Colas }\end{array}$} \\
\hline & & & & \multicolumn{2}{|c|}{$\begin{array}{c}95 \% \text { Intervalo de } \\
\text { Confianza }\end{array}$} & & & \\
\hline & & & & Inferior & Superior & & & \\
\hline Par 1 &,- 7826 & 2,50375 &, 52207 & -18653 &, 3001 & $-1,499$ & 22 & ,148 \\
\hline Par 2 & 14,6957 & 6,09347 & 1,27058 & 17,330 & 12,06 & $-11,56$ & 22 &, 000 \\
\hline
\end{tabular}

Para esta variable podemos observar cómo entre los dos pretest no hay evidencias para rechazar la hipótesis nula de igualdad de medias mientras que entre el segundo pretest y el postest las diferencias son significativas al $99 \%$.

\section{Análisis de la variable: Originalidad Verbal}

También aquí las diferencias entre grupos en el postest han resultado estadísticamente significativas $(\mathrm{F}=158,071 ; \mathrm{p}<0.001)$. Igualmente lo fueron las diferencias entre segundo pretest y postest para el grupo expe$\operatorname{rimental}(\mathrm{t}=-14.753 ; \mathrm{p}<0.001)$.

El Análisis de Covarianza realizado en los resultados del postest mostró lo siguiente:

Tabla 10.

\begin{tabular}{|c|c|c|c|c|c|}
\hline Fuente & Suma de Cuadrados & Gl & $\begin{array}{c}\text { Desviaciones Cua- } \\
\text { dráticas Medias } \\
\end{array}$ & $\mathrm{F}$ & Sig. \\
\hline Modelo Corregido & 7217,066 & 3 & 2405,689 & 144,27 & 2,000 \\
\hline Constante & 207,254 & 1 & 207,254 & $12^{\prime} 42$ & ,001 \\
\hline GRUPO & 5271,645 & 2 & 2635,823 & $\mathbf{1 5 8 , 0 7}$ &, 000 \\
\hline $\mathrm{O}_{-} \mathrm{V}_{-} \mathrm{Y}$ & 949,312 & 1 & 949,312 & $56^{\prime} 93$ &, 000 \\
\hline Error & 983'823 & 59 & 16,675 & & \\
\hline Total & 56812,000 & 63 & & & \\
\hline Total Corregido & 8200,889 & 62 & & & \\
\hline
\end{tabular}

$R$ Cuadrado $=, 880$ ( $R$ Cuadrado Ajustado $=, 874)$

Se rechaza la hipótesis de igualdad de medias en los distintos grupos.

Tabla 11. ANOVA de ganancias

\begin{tabular}{|c|c|c|c|c|c|}
\hline Fuente & Suma de Cuadrados & $\mathrm{gl}$ & $\begin{array}{c}\text { Desviaciones } \\
\text { Cuadráticas Me- } \\
\text { dias } \\
\end{array}$ & $\mathrm{F}$ & Sig. \\
\hline Modelo Corregido & 6267,755 & 2 & 3133,877 & 97,268 & ,000 \\
\hline Constante & 45983,249 & 1 & 45983,249 & 1427,213 &, 000 \\
\hline GRUPO & 6267,755 & 2 & $\mathbf{3 1 3 3 , 8 7 7}$ & 97,268 &, 000 \\
\hline Error & 1933,134 & 60 & 32,219 & & \\
\hline Total & 56812,000 & 63 & & & \\
\hline Total Corregido & 8200,889 & 62 & & & \\
\hline
\end{tabular}

R Cuadrado $=, 764$ ( $R$ Cuadrado Ajustado = ,756)

Se confirman diferencias significativas por grupos.

Tabla 12. 
Franco Justo, C. (2004). Aplicación de un programa psicoeducativo para formentar la creatividad en la etapa de educación infantil. RELIEVE, v. 10, n. 2, p. 245-266.

http://www.uv.es/RELIEVE/v10n2/RELIEVEv10n2 4.htm

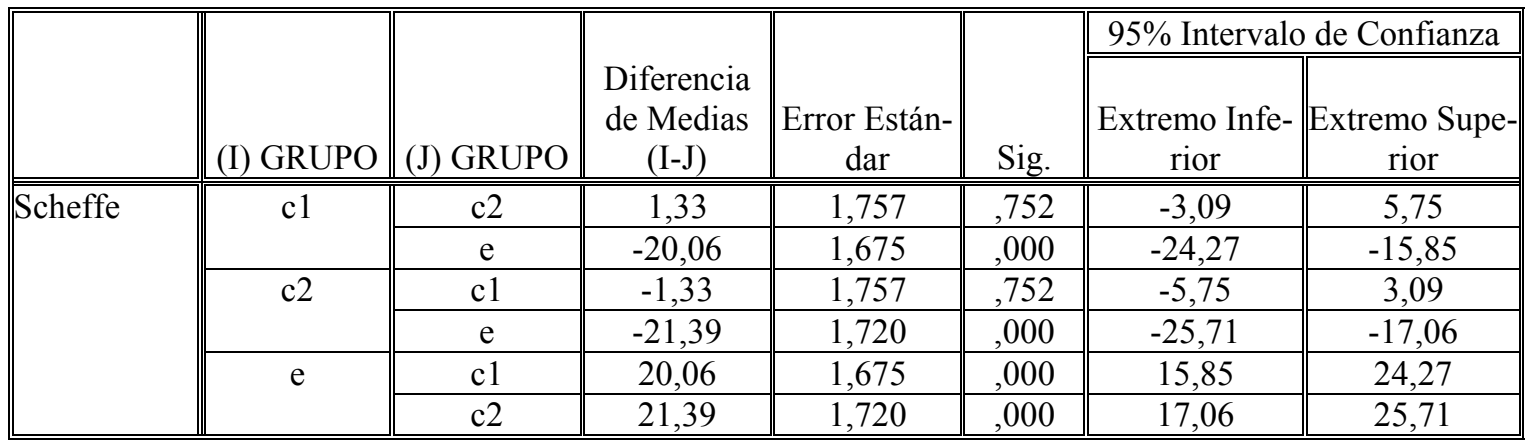

Nuevamente el test empleado confirma la existencia de diferencias significativas entre los grupos control y el grupo experimental y

no evidencia diferencias significativas entre los dos grupos control.

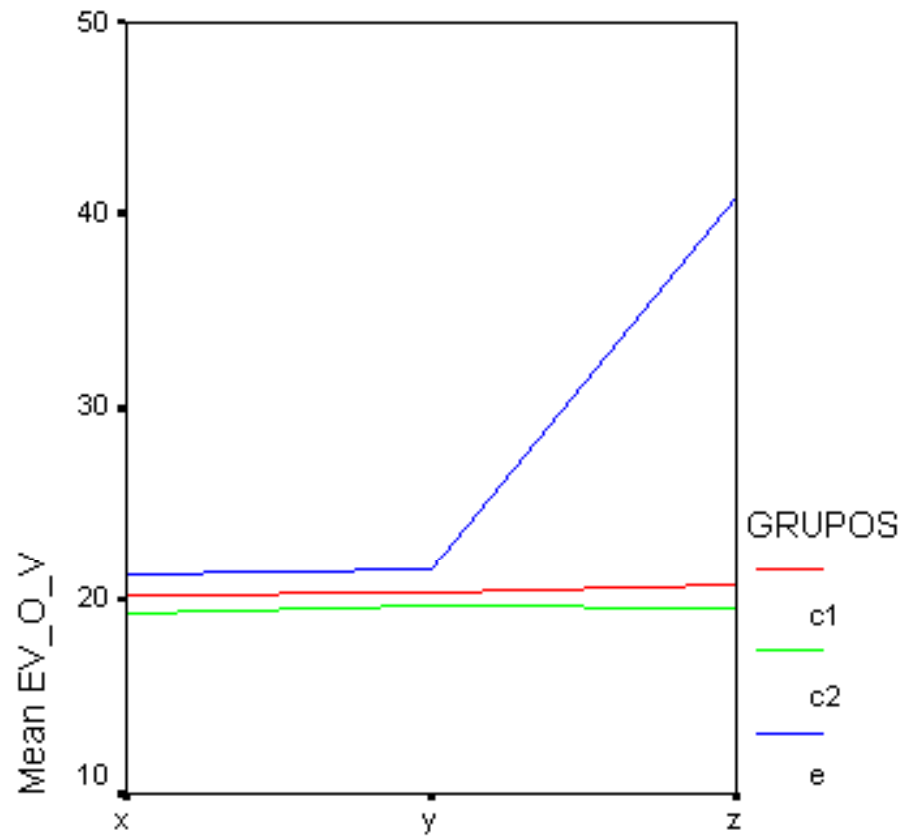

PERIODOS

Aunque gráficamente resulte evidente, vamos a analizar separadamente los datos del grupo experimental en busca de diferencias entre los tres momentos de la medición:

Tabla 13.

\begin{tabular}{|c|c|c|c|c|c|c|c|c|}
\hline & \multirow{3}{*}{ Media } & \multirow{3}{*}{$\begin{array}{c}\text { Desviación } \\
\text { Típica }\end{array}$} & \multirow{3}{*}{$\begin{array}{c}\text { Error } \\
\text { Estándar }\end{array}$} & & & \multirow{3}{*}{$\mathrm{t}$} & \multirow{3}{*}{ gl } & \multirow{3}{*}{$\begin{array}{l}\text { Nivel de Sig- } \\
\text { nificación a } 2 \\
\text { Colas }\end{array}$} \\
\hline & & & & \multicolumn{2}{|c|}{$\begin{array}{l}95 \% \text { Intervalo de } \\
\text { Confianza }\end{array}$} & & & \\
\hline & & & & Inferior & Superior & & & \\
\hline Par 1 &,- 3043 & 1,66337 & ,34684 & $-1,023$ &, 4149 &,- 877 & 22 & ,390 \\
\hline Par 2 & 19,2609 & 6,26112 & 1,30553 & $-21,96$ & $-16,55$ & $-14,75$ & 22 &, 000 \\
\hline
\end{tabular}

Como en la variable anterior, aquí tampoco se detectan diferencias significativas en el

\section{Creatividad Gráfica}

primer par y sí en el segundo con un error de un $1 \%$. 
Franco Justo, C. (2004). Aplicación de un programa psicoeducativo para formentar la creatividad en la etapa de educación infantil. RELIEVE, v. 10, n. 2, p. 245-266.

http://www.uv.es/RELIEVE/v10n2/RELIEVEv10n2 4.htm

Tabla 14

\begin{tabular}{|c|c|c|c|c|c|}
\hline VARIABLE & GRUPO & MEDIDA & MEDIA & D.T. & C.V. \\
\hline \multirow{9}{*}{ Flexibilidad } & \multirow{3}{*}{ Control 1} & Pretest 1 & 12.29 & 2.918 & 0.237 \\
\hline & & Pretest 2 & 12.95 & 2.924 & 0.226 \\
\hline & & Postest & 13.14 & 3.054 & 0.232 \\
\hline & \multirow{3}{*}{ Control 2} & Pretest 1 & 13.21 & 2.070 & 0.157 \\
\hline & & Pretest 2 & 13.26 & 2.104 & 0.159 \\
\hline & & Postest & 14.16 & 2.062 & 0.146 \\
\hline & \multirow{3}{*}{ Experimental } & Pretest 1 & 12.52 & 3.342 & 0.267 \\
\hline & & Pretest 2 & 12.52 & 3.260 & 0.260 \\
\hline & & Postest & 15.26 & 4.147 & 0.272 \\
\hline \multirow{9}{*}{ Fluidez } & \multirow{3}{*}{ Control 1} & Pretest 1 & 12.86 & 2.242 & 0.174 \\
\hline & & Pretest 2 & 13.19 & 2.562 & 0.194 \\
\hline & & Postest & 14.14 & 2.869 & 0.203 \\
\hline & \multirow{3}{*}{ Control 2} & Pretest 1 & 13.84 & 2.141 & 0.155 \\
\hline & & Pretest 2 & 13.79 & 1.988 & 0.144 \\
\hline & & Postest & 14.11 & 1.853 & 0.131 \\
\hline & \multirow{3}{*}{ Experimental } & Pretest 1 & 13.13 & 2.685 & 0.204 \\
\hline & & Pretest 2 & 13.87 & 2.897 & 0.209 \\
\hline & & Postest & 16.43 & 3.287 & 0.200 \\
\hline \multirow{9}{*}{ Originalidad } & \multirow{3}{*}{ Control 1} & Pretest 1 & 14.29 & 3.810 & 0.267 \\
\hline & & Pretest 2 & 14.52 & 3.628 & 0.250 \\
\hline & & Postest & 15.33 & 3.230 & 0.211 \\
\hline & \multirow{3}{*}{ Control 2} & Pretest 1 & 15.74 & 3.364 & 0.214 \\
\hline & & Pretest 2 & 16.53 & 3.373 & 0.204 \\
\hline & & Postest & 16.63 & 3.547 & 0.213 \\
\hline & \multirow{3}{*}{ Experimental } & Pretest 1 & 14.52 & 3.964 & 0.273 \\
\hline & & Pretest 2 & 14.91 & 3.789 & 0.254 \\
\hline & & Postest & $\mid 17.61$ & $\mid 3.590$ & 0.204 \\
\hline
\end{tabular}

La dispersión de los datos referentes al aspecto gráfico de la creatividad se mantiene también baja y oscila entre un valor de 0.131 del $2^{\circ}$ grupo control en la medida postest en cuanto a la variable fluidez gráfica y 0.273 del grupo experimental en el primer pretest en cuanto a la variable originalidad. Podemos apreciar también medias más altas en el grupo experimental que en los dos control, aunque en general no parecen subir tanto como las variables de creatividad verbal.

\section{Análisis de la variable: Flexibilidad Grá- fica}

En esta variable también se obtuvieron diferencias significativas entre los distintos grupos $(F=14,429 ; p<0.001)$, y al analizar los datos del grupo experimental y comparar la segunda medida pretest y la postest $(\mathrm{t}=$ 6.274; $\mathrm{p}<0.001)$.

El Análisis de Covarianza realizado se muestra en la siguiente tabla: 
Franco Justo, C. (2004). Aplicación de un programa psicoeducativo para formentar la creatividad en la etapa de educación infantil. RELIEVE, v. 10, n. 2, p. 245-266.

http://www.uv.es/RELIEVE/v10n2/RELIEVEv10n2 4.htm

Tabla 15.

\begin{tabular}{|c|c|c|c|c|c|}
\hline Fuente & Suma de Cuadrados & $\mathrm{gl}$ & $\begin{array}{c}\text { Desviaciones } \\
\text { Cuadráticas } \\
\text { Medias } \\
\end{array}$ & $\mathrm{F}$ & Sig. \\
\hline Modelo Corregido & 535,428 & 3 & 178,476 & 67,735 & ב,000 \\
\hline Constante & 4,353 & 1 & 4,353 & 1,652 & ,204 \\
\hline GRUPO & 76,038 & 2 & 38,019 & 14,429 &, 000 \\
\hline FLE_M_Y & 486,072 & 1 & 486,072 & 184,472 &, 000 \\
\hline Error & 155,461 & 59 & 2,635 & & \\
\hline Total & 13434,000 & 63 & & & \\
\hline Total Corregido & 690,889 & 62 & & & \\
\hline
\end{tabular}

Como podemos, se rechaza la hipótesis de igualdad de medias en los distintos grupos, por lo que el contraste resulta significativo.

Tabla 16. ANOVA

\begin{tabular}{|c|c|c|c|c|c|}
\hline Fuente & Suma de Cuadrados & $\mathrm{gl}$ & \begin{tabular}{|c|} 
Desviaciones \\
Cuadráticas Me- \\
dias
\end{tabular} & $\mathrm{F}$ & Sig. \\
\hline Modelo Corregido & 49,356 & 2 & 24,678 & 2,308 & , 108 \\
\hline Constante & 12603,532 & 1 & 12603,532 & 1178,758 &, 000 \\
\hline GRUPO & 49,356 & 2 & 24,678 & 2,308 &, 108 \\
\hline Error & 641,533 & 60 & 10,692 & & \\
\hline Total & 13434,000 & 63 & & & \\
\hline Total Corregido & 690,889 & 62 & & & \\
\hline
\end{tabular}

$R$ Cuadrado $=, 071$ ( $R$ Cuadrado Ajustado $=, 040)$

Tabla 17

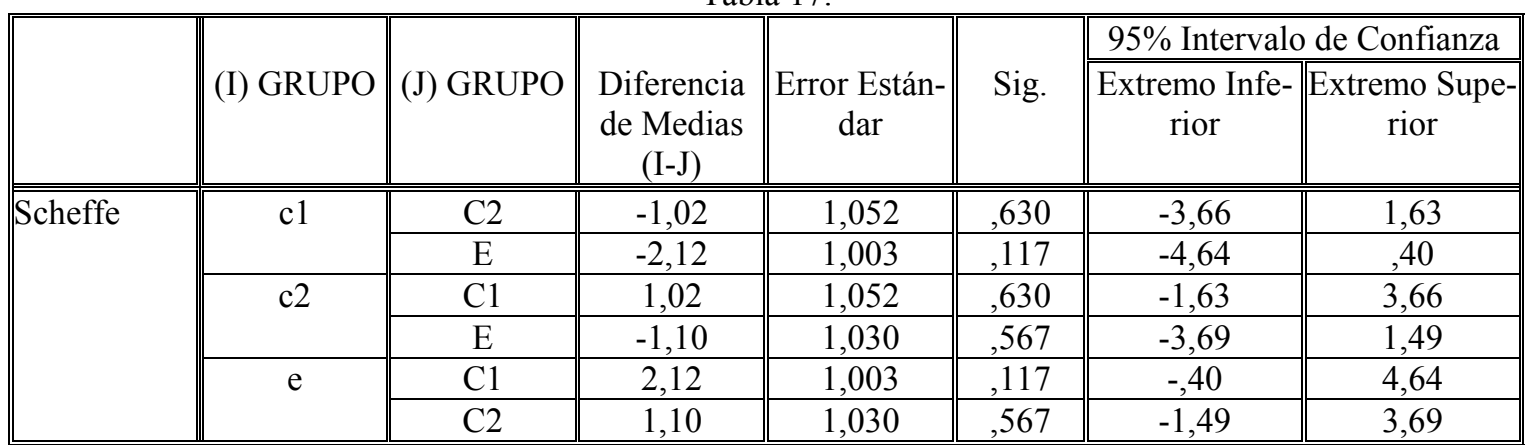


Franco Justo, C. (2004). Aplicación de un programa psicoeducativo para formentar la creatividad en la etapa de educación infantil. RELIEVE, v. 10, n. 2, p. 245-266.

http://www.uv.es/RELIEVE/v10n2/RELIEVEv10n2 4.htm

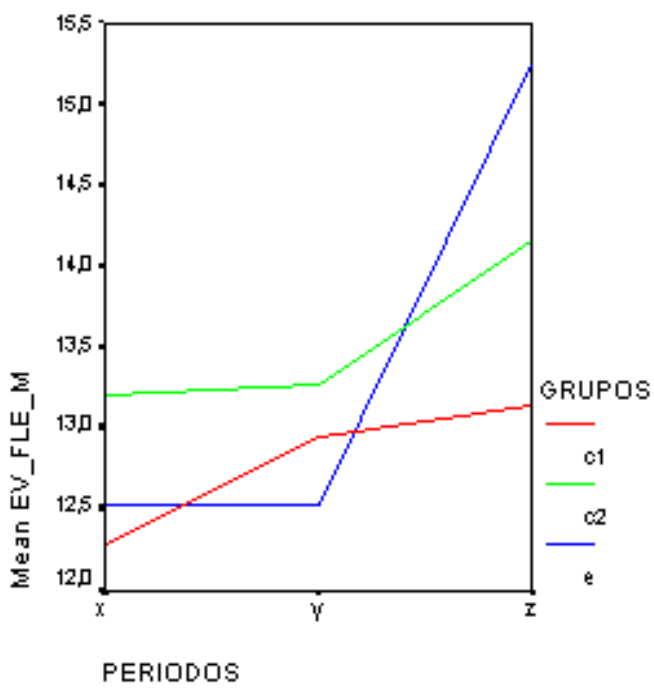

Analizamos para terminar las diferencias tres fases de medición mediante un contraste del grupo experimental consigo mismo en las de medias para muestras apareadas:

Tabla 18.

\begin{tabular}{|c|c|c|c|c|c|c|c|c|}
\hline & \multirow{3}{*}{ Media } & \multirow{3}{*}{$\begin{array}{l}\text { Desviación } \\
\text { Típica }\end{array}$} & \multirow{3}{*}{$\begin{array}{c}\text { Error } \\
\text { Estándar }\end{array}$} & & & \multirow{3}{*}{$\mathrm{t}$} & \multirow{3}{*}{$\mathrm{gl}$} & \multirow{3}{*}{$\begin{array}{l}\text { Nivel de Sig- } \\
\text { nificación a } 2 \\
\text { Colas }\end{array}$} \\
\hline & & & & \multicolumn{2}{|c|}{$\begin{array}{c}95 \% \text { Intervalo de } \\
\text { Confianza } \\
\end{array}$} & & & \\
\hline & & & & Inferior & Superior & & & \\
\hline Par 1 & ,0000 & 1,16775 & ,24349 & -5050 &, 5050 & ,000 & 22 & 1,000 \\
\hline Par 2 & -27391 & 2,09336 & ,43656 & $-3,644$ & $-1,8338$ & $-6,274$ & 22 &, 000 \\
\hline
\end{tabular}

Obtenemos que no hay diferencias significativas entre los dos pretest pero sí las hay con una confianza del 99\% entre el segundo pretest y el postest.

\section{Estudio de la Variable: Fluidez Gráfica}

También aquí se detectan diferencias significativas entre los distintos grupos si eliminamos el efecto que puedan tener los niveles

Tabla 19. ANCOVA

\begin{tabular}{||l||c||c||c||c||c||}
\hline \multicolumn{1}{|c||}{ Fuente } & Suma de Cuadrados & Gl & $\begin{array}{c}\text { Desviaciones Cua- } \\
\text { dráticas Medias }\end{array}$ & F & Sig. \\
\hline \hline Modelo Corregido & 413,090 & 3 & 137,697 & 63,052 &, 000 \\
\hline Constante & 9,978 & 1 & 9,978 & 4,569 &, 037 \\
\hline GRUPO & $\mathbf{5 8 , 4 9 1}$ & $\mathbf{2}$ & $\mathbf{2 9 , 2 4 5}$ & $\mathbf{1 3 , 3 9 2}$ & $\mathbf{0 0 0}$ \\
\hline FLU_M_Y & 335,166 & 1 & 335,1666 & 153,475 &, 000 \\
\hline Error & 128,847 & 59 & 2,184 & & \\
\hline Total & 14657,000 & 63 & & & \\
\hline Total Corregido & 541,937 & 62 & & & \\
\hline
\end{tabular}

de fluidez gráfica previos al experimento ( $\mathrm{F}$ $=13,392 ; \mathrm{p}<0.001)$. También se han encontrado diferencias significativas entre el segundo pretest y la medición postest para el grupo experimental $(\mathrm{t}=-6.145 ; \mathrm{p}<0.001)$. Exponemos a continuación los análisis realizados: 
Franco Justo, C. (2004). Aplicación de un programa psicoeducativo para formentar la creatividad en la etapa de educación infantil. RELIEVE, v. 10, n. 2, p. 245-266.

http://www.uv.es/RELIEVE/v10n2/RELIEVEv10n2 4.htm

Volvemos a obtener diferencias significativas entre los grupos.

Tabla 20. ANOVA

\begin{tabular}{|c|c|c|c|c|c|}
\hline Fuente & Suma de Cuadrados & Gl & $\begin{array}{c}\text { Desviaciones } \\
\text { Cuadráticas } \\
\text { Medias }\end{array}$ & $\mathrm{F}$ & Sig. \\
\hline Modelo Corregido & 77,923 & 2 & 38,962 & "5,038 & ב,009 \\
\hline Constante & 13891,166 & 1 & 13891,166 & 1796 &, 000 \\
\hline GRUPO & 77,923 & 2 & 38,962 & 5,038 &, 009 \\
\hline Error & 464,013 & 60 & 7,734 & & \\
\hline Total & 14657,000 & 63 & & & \\
\hline Total Corregido & 541,937 & 62 & & & \\
\hline
\end{tabular}

$R$ Cuadrado $=, 144($ Cuadrado Ajustado $=, 115)$

En la tabla 21 donde aparece el test de comparaciones múltiples, vemos como éste test refleja la existencia de diferencias significativas entre los grupos control y el grupo experimental aunque no entre los dos grupos control.

Tabla 21. Tests de Comparaciones Múltiples

\begin{tabular}{|c|c|c|c|c|c|c|c|}
\hline & \multirow[b]{2}{*}{ (I) GRUPO } & \multirow[b]{2}{*}{ (J) GRUPO } & \multirow[b]{2}{*}{$\begin{array}{c}\begin{array}{c}\text { Diferencia } \\
\text { de Medias } \\
(\mathrm{I}-\mathrm{J})\end{array} \\
\end{array}$} & \multirow[b]{2}{*}{$\begin{array}{c}\text { Error Están- } \\
\text { dar }\end{array}$} & \multirow[b]{2}{*}{ Sig. } & \multicolumn{2}{|c|}{ 95\% Intervalo de Confianza } \\
\hline & & & & & & $\begin{array}{c}\text { Extremo Infe- } \\
\text { rior }\end{array}$ & $\begin{array}{c}\text { Extremo Supe- } \\
\text { rior } \\
\end{array}$ \\
\hline \multirow[t]{6}{*}{ Scheffe } & \multirow[t]{2}{*}{$\mathrm{C} 1$} & $\mathrm{C} 2$ & ,04 & ,886 & (999 & $-2,19$ & 2,27 \\
\hline & & $\mathrm{E}$ & $-2,29$ & ,845 &, 031 & $-4,42$ &,- 17 \\
\hline & \multirow[t]{2}{*}{$\mathrm{C} 2$} & $\mathrm{C} 1$ &,- 04 & ,886 & ,999 & $-2,27$ & 2,19 \\
\hline & & $\mathrm{E}$ & $-2,33$ & ,868 &, 034 & $-4,51$ &,- 15 \\
\hline & \multirow[t]{2}{*}{$\mathrm{E}$} & $\mathrm{C} 1$ & 2,29 &, 845 &, 031 &, 17 & 4,42 \\
\hline & & $\mathrm{C} 2$ & 2,33 & ,868 &, 034 & 15 & 4,51 \\
\hline
\end{tabular}

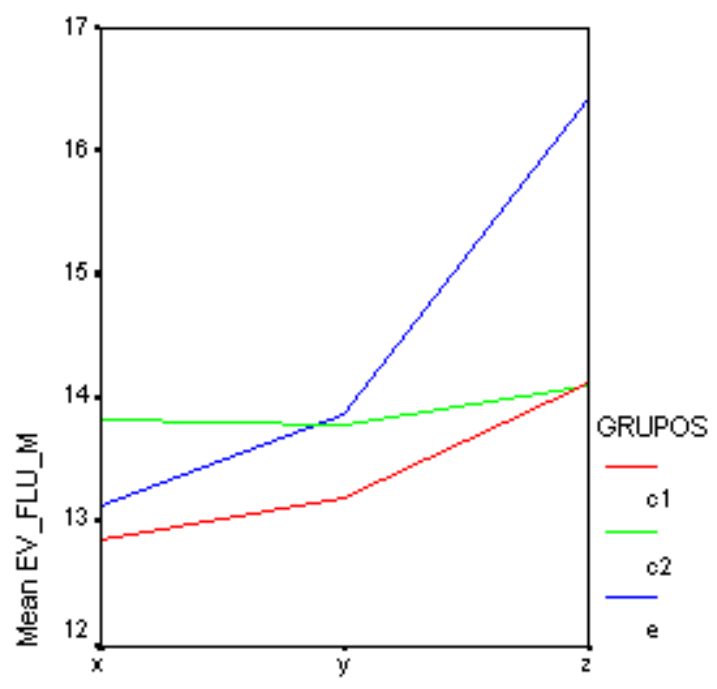

PERIODOS 
Franco Justo, C. (2004). Aplicación de un programa psicoeducativo para formentar la creatividad en la etapa de educación infantil. RELIEVE, v. 10, n. 2, p. 245-266.

http://www.uv.es/RELIEVE/v10n2/RELIEVEv10n2 4.htm

Al analizar la posible existencia de diferencias en las tres mediciones realizadas en el grupo experimental obtuvimos los siguientes

Tabla 22

\begin{tabular}{|c|c|c|c|c|c|c|c|c|}
\hline & & & & & & \multirow{3}{*}{$\mathrm{t}$} & \multirow{3}{*}{$\mathrm{gl}$} & \multirow{3}{*}{$\begin{array}{c}\text { Nivel de Sig- } \\
\text { nificación a } 2 \\
\text { Colas }\end{array}$} \\
\hline & \multirow{2}{*}{ Media } & \multirow{2}{*}{$\begin{array}{l}\text { Desviación } \\
\text { Típica }\end{array}$} & \multirow{2}{*}{$\begin{array}{l}\text { Error } \\
\text { Estándar }\end{array}$} & \multicolumn{2}{|c|}{$\begin{array}{c}95 \% \text { Intervalo de } \\
\text { Confianza }\end{array}$} & & & \\
\hline & & & & Inferior & Superior & & & \\
\hline Par 1 &,- 37 & 1,222 & ,154 &,- 67 & ,21 & $-1,037$ & 22 & 0,096 \\
\hline Par 2 & $-1,35$ & 1,743 & 220 & $-1,79$ &,- 91 & $-6,145$ & 22 &, 000 \\
\hline
\end{tabular}

En este caso no habría diferencias entre las dos medidas pretest y sí entre la segunda y la postest al $99 \%$,

\section{Análisis de la variable: Originalidad Gráfica}

Se han detectado diferencias significativas entre los distintos grupos en la medida postest $(F=14,668 ; p<0.001)$ y también entre el segundo pretest y el postest para el grupo experimental $(\mathrm{t}=-5.811 ; \mathrm{p}<0.001)$.Veamos las tablas y gráficos correspondientes:

Tabla 23. Análisis de Covarianza

\begin{tabular}{|c|c|c|c|c|c|}
\hline Fuente & Suma de Cuadrados & gl & $\begin{array}{c}\text { Desviaciones } \\
\text { Cuadráticas Me- } \\
\text { dias } \\
\end{array}$ & $\mathrm{F}$ & Sig. \\
\hline Modelo Corregido & 638,696 & 3 & 212,899 & 91,781 &, 000 \\
\hline Constante & 35,060 & 1 & 35,060 & 15,114 &, 000 \\
\hline GRUPO & 68,047 & 2 & 34,024 & 14,668 &, 000 \\
\hline O_M_Y & 581,707 & 1 & 581,707 & 250,773 &, 000 \\
\hline Error & 136,859 & 59 & 2,320 & & \\
\hline Total & 18043,000 & 63 & & & \\
\hline Total Corregido & 775,556 & 62 & & & \\
\hline
\end{tabular}

R Cuadrado $=, 824$ ( R Cuadrado Ajustado =,815)

Vemos que hay diferencias significativas entre los grupos con un nivel de significación del 1\%.

Tabla 24. ANOVA.

\begin{tabular}{|c|c|c|c|c|c|}
\hline Fuente & Suma de Cuadrados & $\mathrm{gl}$ & $\begin{array}{c}\text { Desviaciones } \\
\text { Cuadráticas } \\
\text { Medias } \\
\end{array}$ & $\mathrm{F}$ & Sig. \\
\hline Modelo Corregido & $\begin{array}{l}56,990 \\
\end{array}$ & 2 & 28,495 & 2'379 & ב,101 \\
\hline Constante & 17098,460 & 1 & 17098,460 & 1427,715 &, 000 \\
\hline GRUPO & $56^{\prime} 990$ & 2 & 28,495 & 2,379 &, 101 \\
\hline Error & 718,566 & 60 & 11,976 & & \\
\hline Total & 18043,000 & 63 & & & \\
\hline Total Corregido & 775,556 & 62 & & & \\
\hline
\end{tabular}

$R$ Cuadrado $=, 073$ ( $R$ Cuadrado Ajustado $=, 043$ )

Al contrario que para la variable anterior, este análisis no detecta diferencias significativas, sin embargo no es algo a tener en cuenta dado el valor de $\mathrm{R}^{2}$ ajustado. 
Tabla 25. Test de Scheffé.

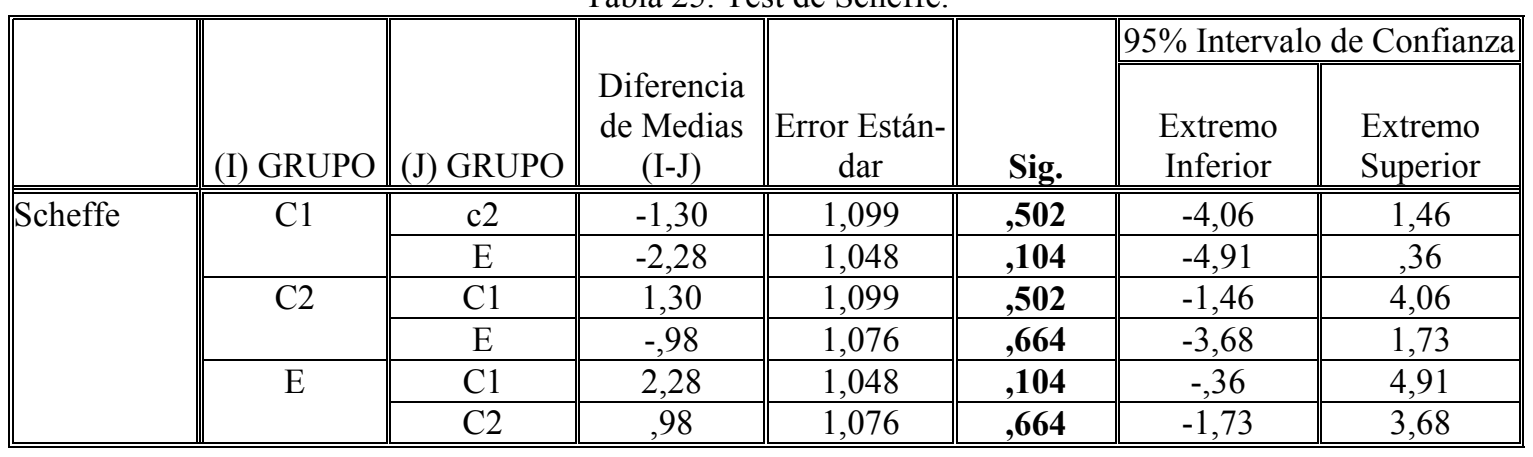

Como podemos observar este test contradice los resultados del ANCOVA no encontrando diferencias significativas entre los distintos grupos en la medida postest. Esta aparente contradicción explica que las medias de la variable dependiente solo difieren realmente al eliminar el efecto de la covaria- da, es decir, si todos los niños partieran de un mismo nivel de originalidad gráfica, veríamos como los del grupo experimental experimentarían un avance significativo en esta variable, tal y como se aprecia en el ANCOVA.

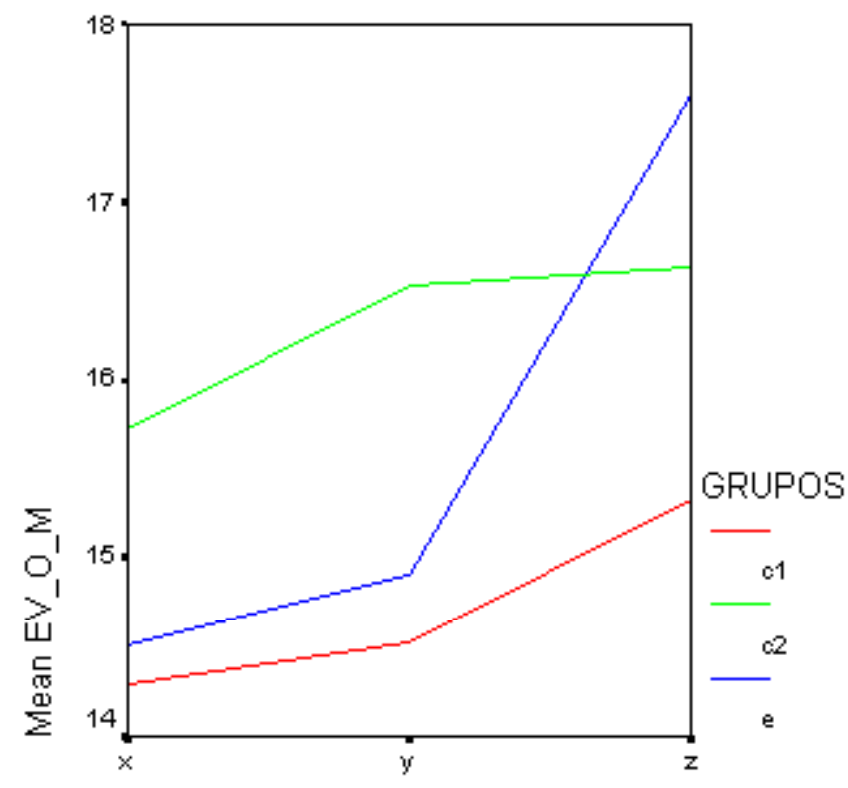

PERIODOS

Al estudiar las diferencias en las medias de esta variable utilizando sólo los datos del grupo experimental en los tres momentos de la medición encontramos que las diferencias entre las dos medidas pretest no son significativas, mientras que entre la segunda medida pretest y la medida postest hay significación asumiendo un error menor al 1\%. 
Tabla 26.

\begin{tabular}{|c|c|c|c|c|c|c|c|c|}
\hline & \multirow{3}{*}{ Media } & \multirow{3}{*}{$\begin{array}{l}\text { Desviación } \\
\text { Típica }\end{array}$} & \multirow{3}{*}{$\begin{array}{c}\text { Error } \\
\text { Estándar }\end{array}$} & & & \multirow{3}{*}{$\mathrm{t}$} & \multirow{3}{*}{ gl } & \multirow{3}{*}{$\begin{array}{l}\text { Nivel de Sig- } \\
\text { nificación a } 2 \\
\text { Colas }\end{array}$} \\
\hline & & & & \multicolumn{2}{|c|}{$\begin{array}{c}95 \% \text { Intervalo de } \\
\text { Confianza }\end{array}$} & & & \\
\hline & & & & Inferior & Superior & & & \\
\hline Par 1 &,- 3913 & 1,23359 & ,25722 &,- 9247 &, 1421 & $-1,521$ & 22 & , 142 \\
\hline Par 2 & $-2,6957$ & 2,22455 & ,46385 & $-3,657$ & $-1,733$ & $-5,811$ & 22 &, 000 \\
\hline
\end{tabular}

\section{DISCUSIÓN}

Una vez analizados estadísticamente los datos obtenidos, hemos comprobado que se ha conseguido el objetivo de esta investigación, a saber, demostrar la factibilidad de mejorar la capacidad creativa de niños/as de 5 años de edad, mediante la utilización de un programa encaminado a ello basado en la utilización del cuento infantil.

Estos datos concuerdan con los obtenidos por otros autores, Ford y Renzulli (1976), Jarial, (1980), Katiyar y Jarial, (1983), Rose y Lin (1984), Torrance y Safter (1986), Amabile (1996), Piqueras (1996), Pérez (2000), y López Martínez (2001), quienes también demostraron con la utilización de diversos programas aplicados en el aula (basados en el juego, en la educación artística, en la resolución de problemas, etc.), la factibilidad de mejorar la producción creativa de los alumnos.

Con respecto al área verbal de la creatividad, se observa que los promedios de las dimensiones de fluidez, flexibilidad y originalidad fueron significativamente mayores en el grupo experimental en relación a los grupos control $(p<0.001)$, en la medición postest. Esto nos lleva a afirmar que la primera hipótesis de nuestro estudio se verifica, ya que hay un aumento significativo en el grupo experimental comparado con los grupos control en sus niveles de creatividad verbal (fluidez, flexibilidad y originalidad).
Con respecto al área gráfica de la creatividad, se puede observar que también existen diferencias significativas entre los grupos en los niveles de flexibilidad, fluidez y originalidad en la medición efectuada en la fase postest, por lo que podemos afirmar que también se cumple la segunda hipótesis de nuestro estudio.

Por tanto, podemos afirmar que, en este trabajo, hemos comprobado de forma empírica las afirmaciones que hacíamos en el marco teórico, al considerar la creatividad como una capacidad susceptible de ser desarrollada y estimulada de acuerdo con las experiencias que el medio proporciona a los sujetos.

En consonancia con lo anterior, estimamos que la consideración de que la creatividad es un talento natural que no puede ser enseñado debe ser revisada a la luz de los resultados obtenidos en esta investigación y otras parecidas.

Esta labor de potenciación y estimulación de las capacidades creativas debe ser asumida de forma principal por el sistema educativo, constituyendo uno de los objetivos principales en función de los cuales debe girar la práctica educativa, ya que el principal objetivo de la educación debe ser el de formar y desarrollar integralmente a las personas.

Dicha labor debe comenzarse desde los primeros niveles, ya que la Educación Infantil es la primera experiencia de educación formal que tiene el niño, la que definirá su 
actitud futura hacia el sistema escolar, y de esta primera experiencia dependerá que se manifieste plenamente, o no, su potencialidad creadora.

Todo esto es importante ya que hoy día se puede constatar que la necesidad de una adecuada adaptación del individuo frente a su medio se está haciendo cada vez más difícil de satisfacer. Este hecho se debe principalmente a la creciente cantidad de demandas y exigencias a las que se ve sometido, y al constante y rápido cambio ya característico de la vida actual. De esta manera, reviste gran importancia la existencia de personas creativas, tanto en el plano científico y artístico, como en el ámbito social y de relaciones humanas (Andreucci y Mayo, 1993).

Por lo tanto, se hace cada vez más necesario contar con personas capaces de adaptarse al medio en el que se desenvuelven. Contar con personas creativas, ya que estas personas presentan un desarrollo más pleno e íntegro de sus capacidades, a la vez que muestran un mayor grado de satisfacción y desarrollo personal.

En este sentido, nos parece de suma importancia desarrollar y estimular el potencial creativo lo más temprano posible en el desarrollo, en el nivel infantil, en donde aún los niños pueden expresarse libremente.

De esta forma, el cuento constituye para los niños una forma de jugar y transformar situaciones conocidas en experiencias novedosas. Además, es especialmente atractivo para estimular la creatividad, porque el pensamiento mágico que presentan los niños en esta etapa, les da más libertad para soñar, creyendo verdaderas las historias, identificándose con los personajes $\mathrm{y}$ atribuyendo características humanas a objetos y animales.

\section{REFERENCIAS BIBLIOGRÁFI- CAS}

Amabile, T. (1996): The social psychology of creativity. New York: Springer-Verlag.
Andreucci, C. y Mayo, B. (1993): El cuento como vehículo para desarrollar la creatividad en preescolares. Tesis Doctoral. Universidad Católica de Chile.

Anguera, M.T.; Arnau, J.; Ato, M.; Martínez, R. ; Pascual, J.; Vallejo, G. (1995): Métodos de investigación en psicología. Madrid: Síntesis.

Delors, J. (1996): La educación encierra un tesoro. Madrid: Santillana.

Ford, B.G. y Renzulli, J.S. (1976): Developing the creative potential of educable mentally retarded students. Journal of Creative Behavior, 10, 210-218.

Gadner, H. (1999): Mentes creativas: una anatomía de la creatividad. Barcelona: Paidós Ibérica.

García Jiménez, (1992): El método experimental en la investigación psicológica. Barcelona: P.P.U.

García, J.F.; Frías, M.D.; Pascual, J. (1999): Los diseños de la investigación experimental. Comprobación de las hipótesis. Valencia: C.S.V.

Jarial, G.S. (1980): An experiment in the training of nonverbal creativity. Journal of Creative Behavior, 14, 270-276.

Katiyar, P.C. y Jarial, G.S. (1983): The role of sex in the enhancement of creativity among adolescents through a processoriented training programme. Indian Educational Review, 19, 40-46.

López Martínez, O. (2001): Evaluación y desarrollo de la creatividad. Tesis Doctoral. Universidad de Murcia.

Madrid, D. (2003): Creatividad, currículum y educación infantil. En A. Gervilla: Creatividad aplicada. Una apuesta de futuro. Madrid: Dykinson.

Marín, R. (1980): La creatividad. Barcelona: CEAC

Moore, C.; Ugarte, M.; Urrutia, E. (1987): Estimulando la creatividad: una experiencia en el sistema educacional chileno. Tesis doctoral. Universidad de Chile.

Pérez Fernández, J.I. (2000): Evaluación de los efectos de un programa de educación artística en la creatividad y en otras varia- 
Franco Justo, C. (2004). Aplicación de un programa psicoeducativo para formentar la creatividad en la etapa de educación infantil. RELIEVE, v. 10, n. 2, p. 245-266.

http://www.uv.es/RELIEVE/v10n2/RELIEVEv10n2 4.htm

bles del desarrollo infantil. Tesis Doctoral. Universidad del País Vasco.

Piqueras Calero, M. (1996): Juego y creatividad. Estudio sobre las posibilidades de fomentar la creatividad artística mediante una enseñanza lúdica. Tesis Doctoral. Universidad de Sevilla.

Raven, J.C. (1966): Test de Matices Progresivas. Barcelona: Paidós.

Repucci, L (1980): Investigaciones sobre la creatividad. En G.A. Davids y J.A. Scott (Edts.): Estrategias para la creatividad. Buenos Aires: Paidós.
Rieben, L. (1979): Inteligencia global, inteligencia operatoria y creatividad. Barcelona: Médica y Técnica.

Rodari, G. (1996): Cuentos por teléfono. Madrid: Editorial Voz de los Sin Voz.

Rose, L.H. y Lin, H. (1984): A meta-analysis of long-term creativity training programs. Journal of Creative Behavior, 18, 11-22.

Torrance, E.P. (1974): Torrance test of creativity thinking. Norms techical manual. Verbal tests, forms $A$ and B; figural tests, forms $A$ and $B$. Personnel Press.

Torrance, E.P. y Safter, H.T. (1986): Are children becoming more creative?. Journal of Creative Behavior, 17, 9-16.

\section{ANEXO 1}

Cuento: Cenicienta

Sesión 1: El Hada Madrina

Objetivos: Desarrollar la fluidez, flexibilidad y originalidad

Modalidad: Grupal

Duración: 70 minutos

Materiales: Hojas en blanco, lápices de colores, botellas de plástico, cajas de cartón, latas, globos, cartulinas, papel de seda, tijeras, pegamento, cinta adhesiva, papel pinocho.

Desarrollo de la sesión: Después de que se les narre el cuento a los niños, se hablará con ellos acerca del Hada Madrina. Para ello, se les preguntará: “¿Cómo se llamaba el Hada Madrina?; ¿Para qué sirve un Hada Madrina?; ¿Qué cosas le pediríais a vuestra Hada madrina?; Y si fuese un Hado Padrino ¿Cómo se hubiese llamado?; ¿Cómo hubiese ido vestido?; ¿Cómo hubiese construido la carroza?". Se les pedirá que dibujen al Hada Madrina y al Hado Padrino.

A continuación, se les dirá a los niños que se imaginen que ellos son unas Hadas Madrinas o Hados Padrinos, y se les preguntará: “¿Para qué otra cosa puede servir una calabaza?; ¿Si no hubieseis tenido una calabaza qué habrías utilizado?; ¿Qué habríais utilizado como caballos?; ¿Y cómo lanceros?".

Finalmente, se dividirá a la clase en grupos de seis, pidiéndoles a cada uno de ellos que hagan una carroza con distintos materiales que encuentren en el aula.

\section{ABOUT THE AUTHORS / SOBRE LOS AUTORES}

Clemente Franco (cfranco@ual.es). Doctor en Psicología. Profesor del Departamento de Psicología Evolutiva y de la Educación de la Universidad de Almería desde el curso 97/98. Sus líneas de investigación se centran, principalmente, en el estudio e intervención de la creatividad y la resiliencia infantil. 
Franco Justo, C. (2004). Aplicación de un programa psicoeducativo para formentar la creatividad en la etapa de educación infantil. RELIEVE, v. 10, n. 2, p. 245-266.

http://www.uv.es/RELIEVE/v10n2/RELIEVEv10n2 4.htm

\section{ARTICLE RECORD / FICHA DEL ARTÍCULO}

\begin{tabular}{|c|c|}
\hline $\begin{array}{l}\text { Reference / } \\
\text { Referencia }\end{array}$ & $\begin{array}{l}\text { Franco, Clemente (2004). Aplicación de un programa psicoeducativo para fomentar la creatividad en la etapa de } \\
\text { educación infantil. Revista ELectrónica de Investigación y EValuación Educativa, v. 10, n. } 2 \text {. } \\
\text { http://www.uv.es/RELIEVE/v10n2/RELIEVEv10n2 } 4 \text {.htmConsultado en (poner fecha). }\end{array}$ \\
\hline Title / Título & $\begin{array}{l}\text { Aplicación de un programa psicoeducativo para fomentar la creatividad en la etapa de educación infantil. [Applying of a } \\
\text { psycho-educative program for developing the creative in the childhood school] }\end{array}$ \\
\hline Authors / Autores & Clemente Franco \\
\hline $\begin{array}{l}\text { Review / } \\
\text { Revista }\end{array}$ & Revista ELectrónica de Investigación y EValuación Educativa (RELIEVE), v. 10, n. 2 \\
\hline ISSN & $1134-4032$ \\
\hline $\begin{array}{l}\text { Publication date / } \\
\text { Fecha de publica- } \\
\text { ción }\end{array}$ & $\begin{array}{l}\text { 2004 (Reception Date: } 2003 \text { Dec 15; Approval Date: } 2004 \text { Oct 10; Publication Date: } 2004 \text { Oct } \\
\text { 21) }\end{array}$ \\
\hline \multirow{2}{*}{$\begin{array}{l}\text { Abstract / } \\
\text { Resumen }\end{array}$} & $\begin{array}{l}\text { The intention of this work is to verify the theoretical assumption which states the relationship be- } \\
\text { tween developing creative potential and the the environmental conditions which the context pro- } \\
\text { vides. A psycho-educational program based on childrens' stories was designed with the aim of } \\
\text { stimulating the creative capability among children in primary school. To measure creativity, the } \\
\text { Torrance Test of Creativity Thinking graphic and verbal battery was used. After applying the pro- } \\
\text { gram, the results showed a huge rise of the levels of verbal creativity in the experimental group } \\
\text { with respect to the control groups.. }\end{array}$ \\
\hline & $\begin{array}{l}\text { En este trabajo, pretendemos comprobar de forma experimental la asunción teórica que plantea la } \\
\text { posibilidad de fomentar el potencial creativo de acuerdo con las condiciones ambientales que el } \\
\text { medio proporciona. Para ello, se diseñó un programa psicoeducativo basado en cuentos infantiles, } \\
\text { con el fin de estimular la capacidad creativa en niños de Educación Infantil. Para la medición de la } \\
\text { creatividad se utilizaron la Batería Gráfica y Verbal de Test de Pensamiento Creativo de Torrance. } \\
\text { Tras la aplicación del programa, los resultados arrojaron un incremento significativo en los niveles } \\
\text { de creatividad verbal en el grupo experimental respecto a los grupos control. }\end{array}$ \\
\hline $\begin{array}{l}\text { Keywords } \\
\text { Descriptores }\end{array}$ & $\begin{array}{l}\text { creativity, children's stories, childhood school. } \\
\text { creatividad, cuento infantil, educación infantil. }\end{array}$ \\
\hline $\begin{array}{l}\text { Institution / } \\
\text { Institución }\end{array}$ & Universidad de Almería (España) \\
\hline $\begin{array}{l}\text { Publication site / } \\
\text { Dirección }\end{array}$ & http://www.uv.es/RELIEVE \\
\hline $\begin{array}{l}\text { Language / } \\
\text { Idioma }\end{array}$ & Español (Title, abstract and keywords in english ) \\
\hline
\end{tabular}

\section{Revista ELectrónica de Investigación y $\mathbf{E V}$ aluación Educativa (RELIEVE)}

[ ISSN: 1134-4032 ]

(C) Copyright, RELIEVE. Reproduction and distribution of this articles it is authorized if the content is no modified and their origin is indicated (RELIEVE Journal, volume, number and electronic address of the document).

(C) Copyright, RELIEVE. Se autoriza la reproducción y distribución de este artículo siempre que no se modifique el contenido y se indique su origen (RELIEVE, volumen, número y dirección electrónica del documento). 